\title{
Interface-Induced Zeeman-Protected Superconductivity in Ultrathin Crystalline Lead Films
}

\author{
Yi Liu, ${ }^{1,2}$ Ziqiao Wang, ${ }^{1,2}$ Xuefeng Zhang, ${ }^{1,2}$ Chaofei Liu, ${ }^{1,2}$ Yongjie Liu, ${ }^{3}$ Zhimou Zhou, ${ }^{1,2}$ \\ Junfeng Wang, ${ }^{3}$ Qingyan Wang, ${ }^{1,2,4}$ Yanzhao Liu, ${ }^{1,2}$ Chuanying Xi, ${ }^{5}$ Mingliang Tian, ${ }^{5}$ \\ Haiwen Liu, ${ }^{6, *}$ Ji Feng, ${ }^{1,2}$ X. C. Xie, ${ }^{1,2}$ and Jian Wang ${ }^{1,2,7, \dagger}$ \\ ${ }^{1}$ International Center for Quantum Materials, School of Physics, Peking University, Beijing 100871, China \\ ${ }^{2}$ Collaborative Innovation Center of Quantum Matter, Beijing 100871, China \\ ${ }^{3}$ Wuhan National High Magnetic Field Center, Huazhong University of Science and Technology, \\ Wuhan 430074, China \\ ${ }^{4}$ Institute of Physics, Chinese Academy of Sciences, Beijing 100190, China \\ ${ }^{5}$ High Magnetic Field Laboratory, Chinese Academy of Sciences, Hefei 230031, Anhui, China \\ ${ }^{6}$ Center for Advanced Quantum Studies, Department of Physics, Beijing Normal University, \\ Beijing 100875, China \\ ${ }^{7}$ CAS Center for Excellence in Topological Quantum Computation, \\ University of Chinese Academy of Sciences, Beijing 100190, China
}

(Received 3 September 2017; revised manuscript received 1 February 2018; published 2 April 2018)

\begin{abstract}
Two-dimensional (2D) superconducting systems are of great importance for exploring exotic quantum physics. The recent development of fabrication techniques has stimulated studies of high-quality singlecrystalline 2D superconductors, where intrinsic properties give rise to unprecedented physical phenomena. Here, we report the observation of Zeeman-type spin-orbit interaction protected superconductivity (Zeeman-protected superconductivity) in 4-monolayer (ML) to 6-ML crystalline $\mathrm{Pb}$ films grown on striped incommensurate $\mathrm{Pb}$ layers on $\mathrm{Si}(111)$ substrates by molecular beam epitaxy. An anomalously large in-plane critical field far beyond the Pauli limit is detected, which can be attributed to the Zeeman-protected superconductivity due to the in-plane inversion symmetry breaking at the interface. Our work demonstrates that, in superconducting heterostructures, the interface can induce Zeeman-type spin-orbit interactions and modulate the superconductivity.
\end{abstract}

DOI: 10.1103/PhysRevX.8.021002

Two-dimensional (2D) superconducting systems have gained considerable interest in condensed matter physics. The development of state-of-the-art film-growth techniques makes it possible to investigate the intrinsic properties of high-quality crystalline superconducting films down to a few atomic layers, such as $\mathrm{Pb}$ and In films on $\mathrm{Si}$ [1-8], Ga films on $\mathrm{GaN}[9,10]$, and $\mathrm{FeSe}$ films on $\mathrm{SrTiO}_{3}[11,12]$. In general, the in-plane critical field $B_{c}$ of ultrathin superconducting films mainly depends on the spin pair breaking effect, namely, the Pauli limit $B_{p}[13,14]$. However, several mechanisms can weaken or eliminate the spin pair breaking effect and enhance $B_{c}$ beyond the Pauli limit. For instance,

\footnotetext{
* Corresponding author. haiwen.liu@bnu.edu.cn

Corresponding author. jianwangphysics@pku.edu.cn
}

Published by the American Physical Society under the terms of the Creative Commons Attribution 4.0 International license. Further distribution of this work must maintain attribution to the author(s) and the published article's title, journal citation, and DOI.
Subject Areas: Condensed Matter Physics,

Nanophysics, Superconductivity in spin-triplet superconductors, the Cooper pairs formed by two electrons with parallel spin orientation are immune to the spin pair breaking effect and hence can easily overcome the Pauli limit [15]. In disordered 2D systems with conventional superconducting pairing, the Pauli limit can be surpassed by spin-orbit scattering, which randomizes the spin orientation and thus weakens spin paramagnetism $[16,17]$. In addition, intrinsic spin-orbit interactions (SOIs) can also give rise to the enhancement of $B_{\mathrm{c}}$. Rashba-type SOI, which is generated from out-of-plane inversion asymmetry, can polarize the spin of the electron to the in-plane direction, but the in-plane critical field $B_{c}$ cannot exceed $\sqrt{2} B_{p}$ [18]. In highly crystalline $2 \mathrm{D}$ superconductors with in-plane inversion symmetry breaking, such as single-layer $\mathrm{NbSe}_{2}$ and $\mathrm{MoS}_{2}$, an extremely large in-plane critical field far beyond the Pauli limit is observed, and it is attributed to Ising superconductivity [19,20,22-24]. In these systems, the broken in-plane inversion symmetry gives rise to Zeeman-type SOI, causing an out-of-plane spin polarization of energy valleys; hence, it protects the superconductivity under a high in-plane magnetic field. To fully understand the general effect of Zeeman-type SOI on 
the in-plane critical field, more experimental and theoretical studies on various $2 \mathrm{D}$ crystalline superconductors beyond TMDs are necessary. Nevertheless, most 2D superconductors naturally preserve in-plane inversion symmetry, where Zeeman-protected superconductivity cannot exist.

Here, we report our observation of interface-induced Zeeman-protected superconductivity in ultrathin crystalline $\mathrm{Pb}$ films epitaxially grown on the striped incommensurate (SIC) phase on a Si(111) substrate. Under a parallel magnetic field, superconductivity survives at high fields far beyond the Pauli limit, suggesting Zeeman-protected superconductivity in ultrathin $\mathrm{Pb}$ films. The temperature dependence of the in-plane critical field qualitatively deviates from Klemm-Luther-Beasley (KLB) theory of the spin-orbit impurity scattering mechanism [16]. Furthermore, $a b$ initio calculations show that the special lattice distortion in the SIC phase can extend to $\mathrm{Pb}$ films, which is very likely to induce Zeeman-type SOI and result in the pronounced spin splitting of the band structure in a $\mathrm{Pb}$ film/SIC system. Based on the microscopic analysis, we find that Zeeman-type SOI largely enhances the in-plane critical field and is quantitatively consistent with our experimental observation.

The atomically flat ultrathin crystalline $\mathrm{Pb}$ films were epitaxially grown on a $\mathrm{Pb}$-induced SIC layer on $\mathrm{Si}(111)$ substrate in an ultrahigh-vacuum molecular beam epitaxy (MBE) chamber. The SIC layer has a thickness of $0.26 \mathrm{~nm}$ and is acquired by deposition of 1.5-monolayer (ML) $\mathrm{Pb}$ on $\mathrm{Si}(111)$ substrate at room temperature and by subsequent annealing at about $573 \mathrm{~K}$ for 30 seconds. A typical scanning tunneling microscopy (STM) image of 4-ML $\mathrm{Pb}$ film is shown in Fig. 1. For ex situ transport measurements, $\mathrm{Pb}$ films with a nominal thickness of 4, 5, and $6 \mathrm{ML}$ were protected and immobilized by a 6-nm amorphous $\mathrm{Si}$ capping layer. The substrate and the protection layer

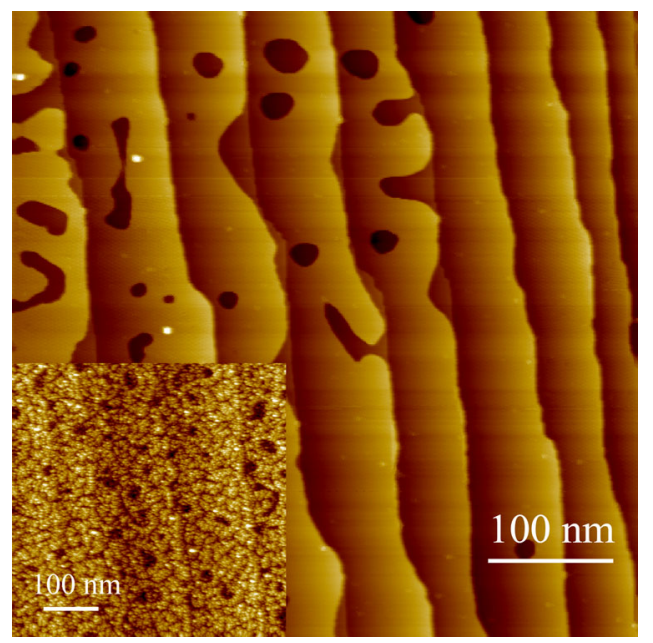

FIG. 1. A typical STM image of 4-ML Pb film $(500 \mathrm{~nm} \times$ $500 \mathrm{~nm}$, sample bias $2.0 \mathrm{~V}$, tunneling current $500 \mathrm{pA}$ ). Inset: STM image of Si capping layer on 4-ML Pb film $(500 \mathrm{~nm} \times 500 \mathrm{~nm}$, sample bias $3.0 \mathrm{~V}$, tunneling current $500 \mathrm{pA}$ ). become insulating below $160 \mathrm{~K}$, providing an ideal platform to study the superconducting properties of $\mathrm{Pb}$ films.

Figure 2 summarizes the transport results of $4-\mathrm{ML} \mathrm{Pb}$ film down to $0.5 \mathrm{~K}$. The schematic of the transport measurement is presented in the inset of Fig. 2(a). According to the temperature-dependent sheet-resistance $R_{\mathrm{sq}}$ curve at zero magnetic field, the superconducting transition occurs at $T_{c}^{\text {onset }}=6.23 \mathrm{~K}$, indicated by extrapolating both the normalstate resistance and the superconducting transition curve [Fig. 2(a)], and the resistance drops to zero within the measurement resolution at $T_{c}^{\text {zero }}=5.78 \mathrm{~K}$. The superconductivity in 4-ML $\mathrm{Pb}$ film is gradually suppressed by increasing perpendicular magnetic fields [Figs. 2(b) and 2(c)]. Moreover, the upper critical field of 4-6-ML $\mathrm{Pb}$ films is plotted in Fig. S2 in Ref. [21], which is well fitted by Werthamer-Helfand-Hohenberg (WHH) theory [25], yielding a larger critical field for thinner film. Figure 2(d) shows the $V(I)$ characteristics measured at various temperatures from $5.8 \mathrm{~K}$ to $6.5 \mathrm{~K}$ in the absence of magnetic field. A power-law dependence of $V \sim I^{\alpha}$ is observed, where $\alpha$ is the slope of $V(I)$ curves plotted in the double-logarithmic scale. The exponent $\alpha$ reduces with increasing temperature and approaches 3 at $6.10 \mathrm{~K}$ [defined as $T_{\mathrm{BKT}}$, see the inset of Fig. 2(d)], indicating a Berezinski-Kosterlitz-Thouless (BKT)-like behavior $[22,26,27]$ in 4-ML Pb film.

Figure 3 shows the main results of the transport measurements on the 4-6-ML $\mathrm{Pb}$ films under a parallel magnetic field. The $R_{\mathrm{sq}}(B)$ curves measured on 4- and 5ML Pb films up to $15 \mathrm{~T}$ are displayed in Figs. 3(a) and 3(b), respectively. A large in-plane critical field (defined as the magnetic field required to reach $50 \%$ of the normal state resistance $R_{\mathrm{n}}$ ) is observed and extrapolated beyond the Pauli limit [the inset of Fig. 3(d)]. To study the critical field behavior at lower temperatures, we carried out a high magnetic field measurement up to $35.5 \mathrm{~T}$ on 6-ML $\mathrm{Pb}$ film [Fig. 3(c)]. The Pauli limit for 6-ML Pb film is estimated by the equation $B_{p}=\Delta_{0} / \sqrt{2} \mu_{B}=14.7 \mathrm{~T}[13,14]$ since $2 \Delta_{0} / k_{B} T_{c}$ remains a constant of about 4.4 for ultrathin crystalline $\mathrm{Pb}$ films [3]. The upper critical field exceeds $35.5 \mathrm{~T}$ at $2.8 \mathrm{~K}$, which is already far beyond the Pauli limit $B_{p}$. Figure 3(d) summarizes the in-plane critical magnetic field $B_{c}$ normalized by $B_{p}$ as a function of reduced temperature $T / T_{\mathrm{c}}$ for 4-6-ML Pb films. Based on the Gor'kov Green's function technique [28], we develop a microscopic model for the Zeeman-protected superconductivity to determine the critical field $B_{c}(T)$ in terms of effective Zeeman-type SOI $\widetilde{\beta_{\mathrm{SO}}}$ :

$$
\begin{aligned}
& \ln \left(\frac{T}{T_{\mathrm{c}}}\right)+\frac{\mu_{B}^{2} B^{2}}{{\widetilde{\beta_{\mathrm{SO}}}}^{2}+\mu_{B}^{2} B^{2}} \operatorname{Re}\left[\psi\left(\frac{1}{2}+\frac{i \sqrt{{\widetilde{\beta_{\mathrm{SO}}}}^{2}+\mu_{B}^{2} B^{2}}}{2 \pi k_{B} T}\right)\right. \\
& \left.-\psi\left(\frac{1}{2}\right)\right]=0
\end{aligned}
$$



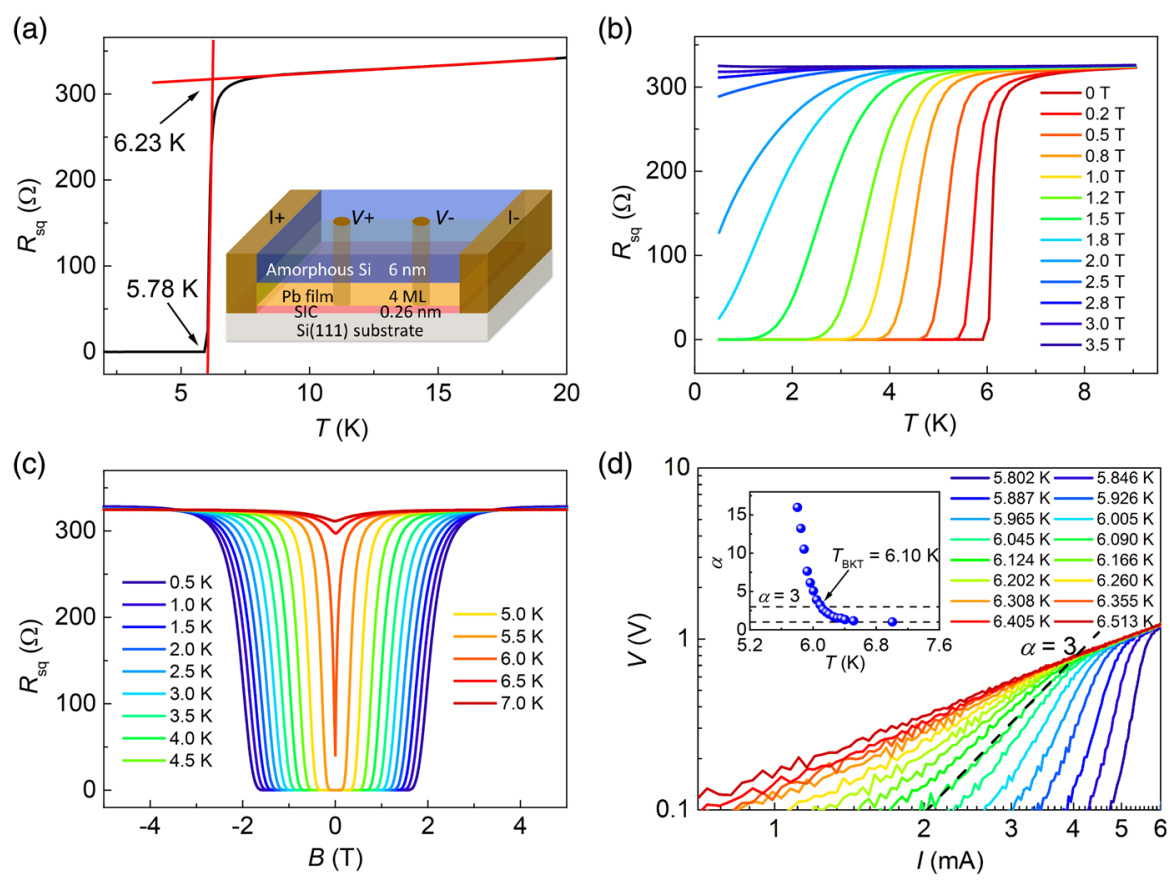

FIG. 2. Transport properties of 4-ML Pb film (sample 1). (a) Temperature dependence of sheet resistance $R_{\mathrm{sq}}$ at zero magnetic field, showing $T_{c}^{\mathrm{onset}}=6.23 \mathrm{~K}$ and $T_{c}^{\text {zero }}=5.78 \mathrm{~K}$. The inset is a schematic for standard four-electrode transport measurements. $(\mathrm{b}) R_{\mathrm{sq}}(\mathrm{T})$ measured under various perpendicular magnetic fields up to $3.5 \mathrm{~T}$. (c) The field dependence of $R_{\mathrm{sq}}$ at different temperatures ranging from 0.5 to $7.0 \mathrm{~K}$. (d) $V(I)$ characteristics at various temperatures from 5.802 to $6.513 \mathrm{~K}$ at $B=0$ T plotted on a double-logarithmic scale. The black line corresponds to $V \propto I^{3}$ dependence. Inset: Power-law exponent $\alpha\left(V \propto I^{\alpha}\right)$ as a function of temperature, showing $T_{\mathrm{BKT}}=6.10 \mathrm{~K}$.

where $\psi(x)$ is the digamma function, $\widetilde{\beta_{\mathrm{SO}}}=\beta_{\mathrm{SO}} /[1+$ $\left.\hbar /\left(2 \pi k_{B} T_{c} \tau_{0}\right)\right]$ is the effective Zeeman-type SOI considering spin-independent scattering, and $\tau_{0}$ is mean free time (more details are shown in Ref. [21]).

The experimental data of $B_{c} / B_{p}$ versus $T / T_{\mathrm{c}}$ can be quantitatively fitted by Eq. (1). The fitting procedure gives effective Zeeman-type SOI $\widetilde{\beta_{\text {SO }}}=3.54 \mathrm{meV}$ and $4.24 \mathrm{meV}$ for 6-ML and 4-ML Pb films. The in-plane critical field $B_{c}$ increases with decreasing film thickness, which yields larger $\widetilde{\beta_{\mathrm{SO}}}$ for thinner films. Since the in-plane inversion symmetry is preserved in both free-standing $\mathrm{Pb}$ atomic layers and Si substrate, the Zeeman-type SOI and large parallel critical field observed in our system should originate from the interface between them.

The SIC phase is a special surface reconstruction of $\mathrm{Pb}$ on $\mathrm{Si}$ (111) substrate with a Pb coverage of 4/3 ML [5]. To be specific, each unit cell of the SIC phase consists of four $\mathrm{Pb}$ atoms (purple circles) located on three surface $\mathrm{Si}$ atoms (blue circles) [Fig. 3(e)]. Three $\mathrm{Pb}$ atoms (marked by 2) form a trimer, which has covalent bonds with the underlying Si substrate. The other one (marked by 1 ) is located at the center of the trimer without bonding to the Si atoms. Metallic bonds are formed within the Pb layer. Distinct from the hexagonal close-packed structure in a freestanding $\mathrm{Pb}$ atomic layer, the $\mathrm{Pb}$ layer in the SIC phase is apparently distorted since the distance between $\mathrm{Pb} 1$ and
$\mathrm{Pb} 3, D_{13}$ (from the nearest unit cell), is about 20\% larger than that between $\mathrm{Pb} 1$ and $\mathrm{Pb} 2, D_{12}$ [Fig. 3(e)] [29]. The special structure of SIC on $\mathrm{Si}(111)$ substrate can give rise to Zeeman-type SOI, which has been demonstrated by DFT calculation [30] and spin-resolved angular-resolved photoemission spectroscopy (ARPES) measurement [31].

The lattice distortion in the SIC phase can extend to the neighboring $\mathrm{Pb}$ layers. Our $a b$ initio calculations show that the similar lattice distortion, evaluated by $D_{13} / D_{12}$, still exists in the neighboring $\mathrm{Pb}$ layers grown on SIC $\left(D_{13}\right.$ is $3.0 \%$ larger than $D_{12}$ for the first Pb layer; see Table $\mathrm{S} 1$ of Ref. [21] for details). Lattice distortion breaks the in-plane inversion symmetry and contributes to the local in-plane crystal field, inducing Zeeman-type SOI in ultrathin lead film on SIC. The spin splitting in Pb film on the SIC phase has been further confirmed by band-structure calculations. We calculate the band structure of 4-ML Pb film on SIC and 5-ML free-standing $\mathrm{Pb}$ film for comparison (Fig. S4), where 5-ML $\mathrm{Pb}$ film can be regarded as a 4-ML Pb film/ SIC system without lattice distortion. Pronounced spin splitting of the energy bands across the Fermi surface is obtained in the 4-ML Pb/SIC system, especially around the $K /-K$ point, which is much larger than that of 5-ML free-standing $\mathrm{Pb}$ film, as expected. Therefore, the spin splitting in the 4-ML Pb/SIC system can be mainly attributed to the lattice distortion and is very likely to be of Zeeman type. Because of the time-reversal symmetry, 


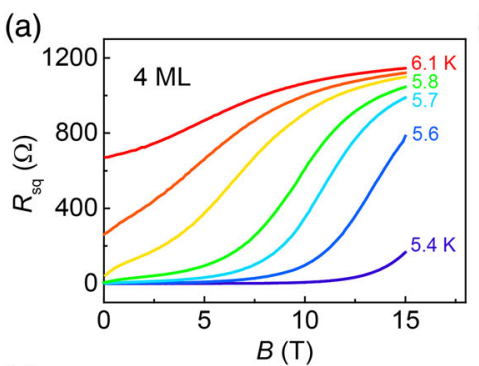

(d)

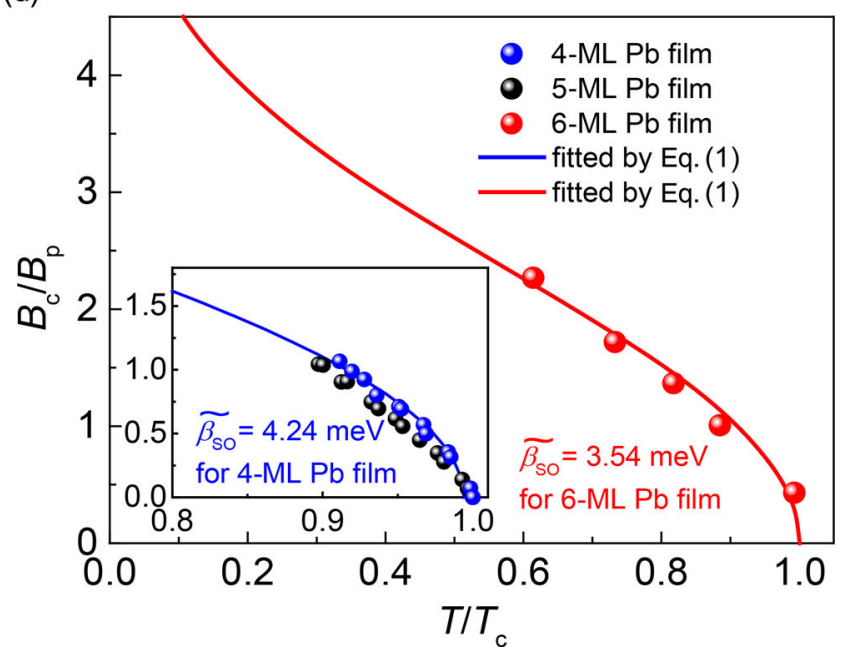

(b)

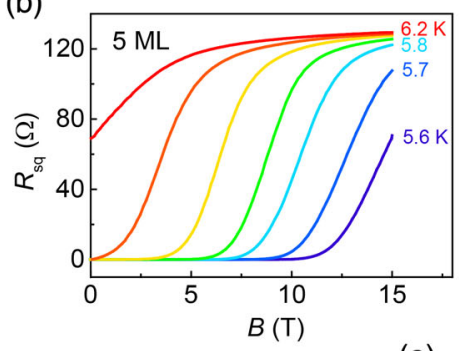

(e)

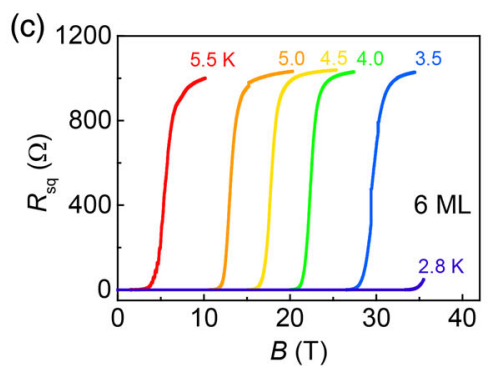

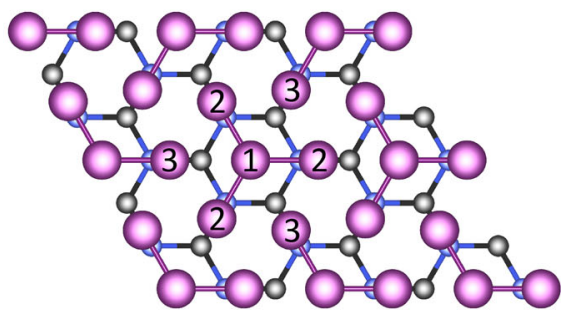

$\mathrm{Pb}$
- First layer Si

O Second layer Si

FIG. 3. Experimental evidence for Zeeman-protected superconductivity in ultrathin (4-6-ML) Pb films. (a)-(c) Parallel magnetic field dependence of $R_{\mathrm{sq}}$ at various temperatures for (a) 4-ML (sample 1), (b) 5-ML (sample 2), and (c) 6-ML (sample 3) Pb films. At 2.8 K or lower temperatures, superconductivity survives under high magnetic fields up to $35.5 \mathrm{~T}$ for 6-ML Pb film. (d) $B_{c}$ normalized by Pauli limit $B_{p}$ as a function of reduced temperature $T / T_{c}$ for 6-ML Pb film. Here, $B_{c}$ is determined as the magnetic field required to reach $50 \%$ of normal-state sheet resistance $R_{n}$. The measured $B_{c}$ can be well fitted by the theoretical curve for Zeeman-protected superconductivity [Eq. (1)], giving effective Zeeman-type SOI $\widetilde{\beta_{\mathrm{SO}}}=3.54 \mathrm{meV}$ in 6-ML Pb film. Inset: Normalized critical field $B_{c} / B_{p}$ for 4-ML and 5-ML $\mathrm{Pb}$ films measured in PPMS with magnetic fields up to $15 \mathrm{~T}$. The blue line is a fitting curve using Eq. (1), giving $\widetilde{\beta_{\mathrm{SO}}}=4.24 \mathrm{meV}$ in 4-ML Pb film. (e) The top view of the crystal structure of the SIC phase. Each unit cell consists of four Pb atoms, with three $\mathrm{Pb}$ atoms (marked as 2) forming a trimer and the other one (marked as 1) located at the center of the trimer. The distance between $\mathrm{Pb} 1$ and $\mathrm{Pb} 3$ (from the nearest unit cell) is much larger than that between $\mathrm{Pb} 1$ and $\mathrm{Pb} 2$, and the in-plane inversion symmetry is broken in this system.

the spin polarization of electrons with momenta at $k$ and $-k$ has opposite direction. Formation of Cooper pairs between electrons with opposite momentum and spin polarizations is thus favored in this situation. Zeeman-type SOI locks the spins of Cooper pairs in the out-of-plane orientation and prevents the alignment of the spin to the external in-plane magnetic field when $\mu_{B} B<\widetilde{\beta_{\mathrm{SO}}}$, and the superconductivity is protected under a large parallel magnetic field far beyond the Pauli limit, i.e., Zeeman-protected superconductivity (Fig. S5).

To further confirm and investigate Zeeman-protected superconductivity in ultrathin crystalline lead film, we measure the $R_{\mathrm{sq}}(B)$ curves of a 6-ML sample in a pulsed parallel magnetic field up to $47 \mathrm{~T}$ [Fig. 4(a)]. The superconductivity survives under a high parallel magnetic field of $40 \mathrm{~T}$ at $1.7 \mathrm{~K}$. In Fig. 4(b), the temperature dependence of an in-plane critical magnetic field, defined as the field corresponding to $50 \% \mathrm{R}_{n}$, is fitted by Eq. (1) with effective
Zeeman-type SOI $\widetilde{\beta_{\text {SO }}}=3.01 \mathrm{meV}$ [close to the value measured in a steady high field for another 6-ML sample, as shown in Fig. 3(d)], suggesting that Zeeman-protected superconductivity is the underlying reason for the enhancement of $B_{\mathrm{c}}$ in 6-ML $\mathrm{Pb}$ film.

We then discuss other possible origins that may contribute to a high parallel critical field in 2D superconductors, such as spin-triplet pairing, spin-orbit impurity scattering, and Rashba-type SOI. In ultrathin crystalline $\mathrm{Pb}$ films, the out-of-plane critical field $B_{\mathrm{c} 2}(T)$ can be well fitted by WHH theory, indicating conventional $s$-wave pairing (Fig. S2). Thus, the contribution from potential spin-triplet pairing due to SOI can be neglected. The microscopic KLB theory [16] points out that the effect of spin paramagnetism can be reduced by spin-orbit impurity scattering, and hence, the parallel critical field can be enhanced beyond the Pauli limit. The KLB formula, however, cannot describe the observed relationship 

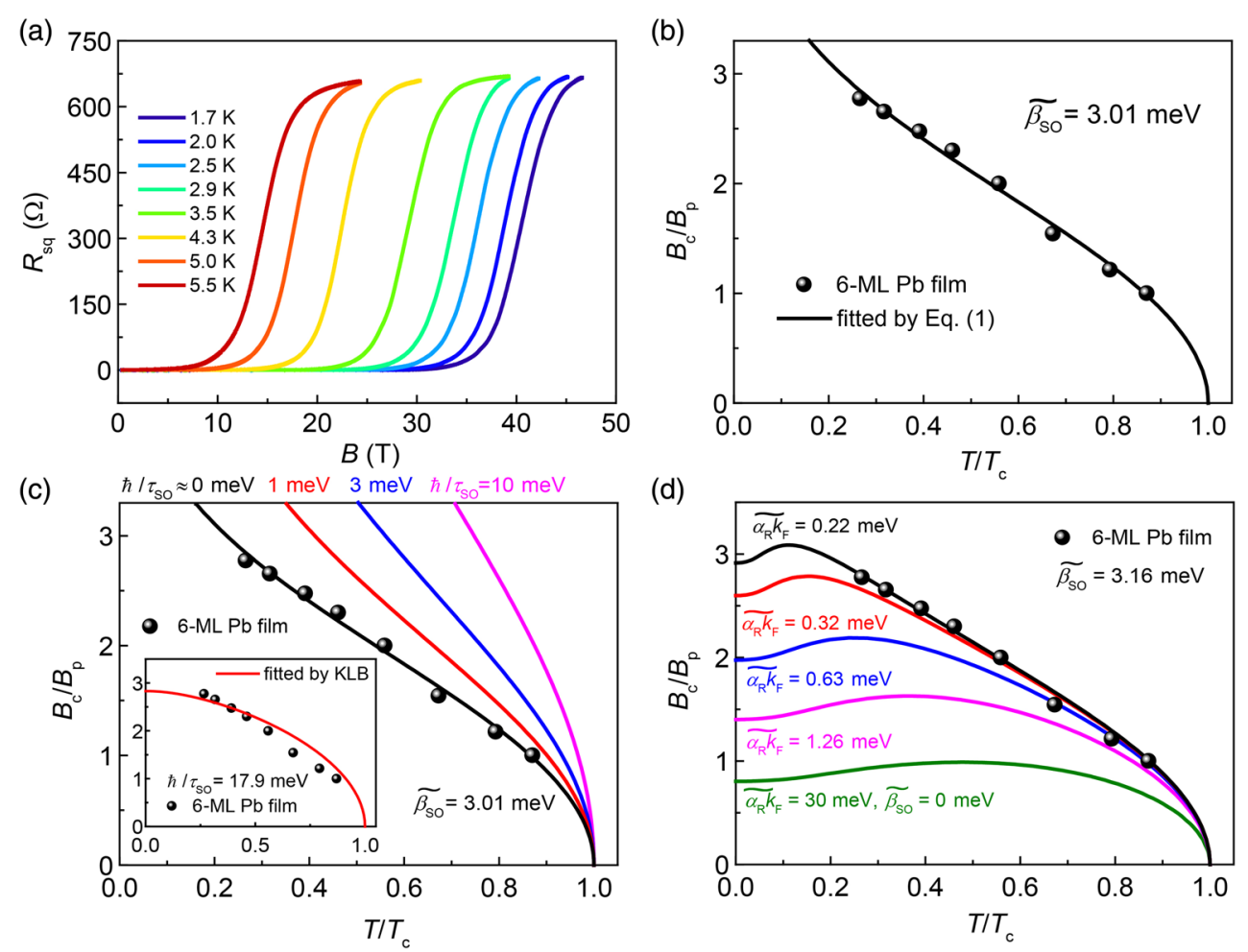

FIG. 4. Pulsed high magnetic field measurement for another 6-ML Pb film (sample 4). (a) $R_{\mathrm{sq}}(B)$ curves at various temperatures measured at parallel magnetic fields up to $47 \mathrm{~T}$. The superconductivity survives under a high parallel magnetic field of $40 \mathrm{~T}$ at $1.7 \mathrm{~K}$. (b) The in-plane critical field $B_{c} / B_{p}$ as a function of normalized temperature $T / T_{c}$ for 6-ML Pb film, showing good agreement with the microscopic theory of Zeeman-protected superconductivity [black solid line, Eq. (1)] and yielding an effective Zeeman-type SOI $\widetilde{\beta_{\mathrm{SO}}}=3.01 \mathrm{meV}$. (c) The $B_{c} / B_{p}\left(T / T_{c}\right)$ curve is fitted based on a microscopic theory considering both effective Zeeman-type SOI and spin-orbit scattering [black solid line, Eq. (2)], and it gives $\hbar / \tau_{\mathrm{SO}} \approx 0 \mathrm{meV}$. The red, blue, and magenta lines indicate the theoretical curves with a fixed $\widetilde{\beta_{\mathrm{SO}}}=3.01 \mathrm{meV}$ and increasing $\hbar / \tau_{\mathrm{SO}}$ from 1 to $10 \mathrm{meV}$. Inset: The relationship between $B_{c} / B_{p}$ and $T / T_{c}$ cannot be fitted by the KLB formula, which describes the in-plane critical field enhanced only by spin-orbit impurity scattering. (d) Theoretical fitting of $B_{c} / B_{p}$ as a function of $T / T_{c}$ using a fixed effective Zeeman-type SOI and increasing effective Rashba-type SOI. The black curve presents the best fit of the experimental data with effective Zeeman-type SOI $\widetilde{\beta_{\mathrm{SO}}}=3.16$ meV and effective Rashba-type SOI $\widetilde{\alpha_{R} k_{F}}=0.22 \mathrm{meV}$. The olive line indicates a special case with only effective Rashba-type SOI $\left(\widetilde{\alpha_{R} k_{F}}=30 \mathrm{meV}, \widetilde{\beta_{\mathrm{SO}}}=0\right)$, which is far below the experimental results.

between $B_{c} / B_{p}$ and $T / T_{c}$ [inset of Fig. 4(c)], indicating that spin-orbit impurity scattering alone cannot account for the observed large $B_{c}$. We develop a microscopic theory considering both Zeeman-type SOI and spin-orbit scattering (the microscopic derivation is given in Ref. [21]):

$$
\begin{aligned}
& \ln \left(\frac{T}{T_{c}}\right)+2 \cdot \operatorname{Re}\left\{\frac { ( \mu _ { B } B ) ^ { 2 } } { ( x _ { 2 } - x _ { 1 } ) ( x _ { 2 } - x _ { 3 } ) } \left[\psi\left(\frac{1}{2}-\frac{x_{1}}{2 \pi k_{B} T}\right)\right.\right. \\
& \left.\left.-\psi\left(\frac{1}{2}-\frac{x_{2}}{2 \pi k_{B} T}\right)\right]\right\}=0
\end{aligned}
$$

where $\psi(x)$ is the digamma function, and $x_{1}, x_{2}, x_{3}$ is the solution of the following equations:

$$
\begin{aligned}
& x^{3}+\frac{2}{3} \frac{\hbar}{\tau_{\mathrm{SO}}} x^{2}+\left(\widetilde{\beta_{\mathrm{SO}}}{ }^{2}+\mu_{B}^{2} B^{2}\right) x+\frac{2}{3} \frac{\widetilde{\hbar \beta_{\mathrm{SO}}}}{\tau_{\mathrm{SO}}}=0 \\
& \operatorname{Im}\left(x_{1}\right)=0
\end{aligned}
$$

with effective Zeeman-type SOI $\widetilde{\beta_{\mathrm{SO}}}=\beta_{\mathrm{SO}} /[1+$ $\left.\hbar /\left(2 \pi k_{B} T_{c} \tau_{0}\right)\right]$ and mean free time $\tau_{\mathrm{SO}}$ for spin-orbit impurity scattering and mean free time $\tau_{0}$ for spinindependent impurity scattering. The fitting curve gives $\hbar / \tau_{\mathrm{SO}} \approx 0 \mathrm{meV}$, which is very small compared to the effective Zeeman-type SOI $\widetilde{\beta_{\text {SO }}}=3.01 \mathrm{meV}$. Therefore, the Zeeman-type SOI is the dominating mechanism of the enhanced $B_{c}$.

Figure 4(d) presents the fitting results of the in-plane critical field $B_{\mathrm{c}}(\mathrm{T})$ data for 6-ML Pb film by a microscopic model with both the Zeeman-type and Rashba-type SOI (see more details and microscopic derivation in Ref. [21]), 
in which $B_{c}$, as a function of $T / T_{c}$, is determined by both the effective Rashba-type SOI $\widetilde{\alpha_{R} k_{F}}$ and Zeeman-type SOI $\widetilde{\beta_{\mathrm{SO}}}$ :

$$
\begin{aligned}
& \ln \left(\frac{T}{T_{c}}\right)+\frac{1}{2}\left[1-\frac{2\left(\widetilde{\alpha_{R} k_{F}}\right)^{2}+\widetilde{\beta_{\mathrm{SO}}}-\mu_{B}^{2} B^{2}}{\rho_{+}^{2}-\rho_{-}^{2}}\right] \\
& \quad \times \operatorname{Re}\left[\psi\left(\frac{1}{2}+\frac{i \rho_{+}}{2 \pi k_{B} T}\right)-\psi\left(\frac{1}{2}\right)\right] \\
& \quad+\frac{1}{2}\left[1+\frac{2\left(\widetilde{\alpha_{R} k_{F}}\right)^{2}+\widetilde{\beta_{\mathrm{SO}}}-\mu_{B}^{2} B^{2}}{\rho_{+}^{2}-\rho_{-}^{2}}\right] \\
& \quad \times \operatorname{Re}\left[\psi\left(\frac{1}{2}+\frac{i \rho_{-}}{2 \pi k_{\mathrm{B}} T}\right)-\psi\left(\frac{1}{2}\right)\right]=0,
\end{aligned}
$$

where $\psi(x)$ is the digamma function, and the arguments are defined as

$$
\begin{aligned}
2 \rho_{ \pm} \equiv & \sqrt{\left(\mu_{B} B+\widetilde{\alpha_{R} k_{F}}\right)^{2}+\left(\widetilde{\alpha_{R} k_{F}}\right)^{2}+\widetilde{\beta_{\mathrm{SO}}^{2}}} \\
& \pm \sqrt{\left(\mu_{B} B-\widetilde{\alpha_{R} k_{F}}\right)^{2}+\left(\widetilde{\alpha_{R} k_{F}}\right)^{2}+{\widetilde{\beta_{\mathrm{SO}}}}^{2}}
\end{aligned}
$$

with effective Zeeman-type SOI $\widetilde{\beta_{\text {SO }}}=\beta_{\text {SO }} /$ $\left[1+\hbar /\left(2 \pi k_{B} T_{c} \tau_{0}\right)\right]$ and effective Rashba-type SOI $\widetilde{\alpha_{R} k_{F}}=\alpha_{R} k_{F} / \sqrt{2}\left[1+\hbar /\left(2 \pi k_{B} T_{c} \tau_{0}\right)\right]$. The result of Eq. (3) is consistent with the previous theory obtained under the clean limit [19].

As shown in Fig. 4(d), the theoretical curve is in good agreement with the experimentally observed in-plane critical field (normalized $B_{c} / B_{p}$ versus $T / T_{c}$ ), with $\widetilde{\beta_{\mathrm{SO}}}=$ $3.16 \mathrm{meV}$ and $\widetilde{\alpha_{R} k_{F}}=0.22 \mathrm{meV}$ for $6-\mathrm{ML} \mathrm{Pb}$ film. In Zeeman-protected superconducting systems, the spin orientation is polarized to the out-of-plane direction by Zeeman-type SOI, while Rashba-type SOI weakens the Zeeman-protection mechanism by tilting the spin of the electron to the in-plane direction, which can be more easily affected by the parallel magnetic field. To clarify the influence of Rashba-type SOI, we display a set of theoretical curves with a fixed effective Zeeman-type SOI $\widetilde{\beta_{\mathrm{SO}}}$ of $3.16 \mathrm{meV}$ and increasing effective Rashba-type SOI $\widetilde{\alpha_{\mathrm{R}} k_{\mathrm{F}}}$ from $0.22 \mathrm{meV}$ to $1.26 \mathrm{meV}$ [Fig. 4(d)]. The Rashbatype SOI bends the curves down at low temperatures and gives rise to a relatively small in-plane critical field, which qualitatively deviates from the quasilinear temperature dependence of our measured data. A special case with only large effective Rashba-type SOI is also considered (olive line, $\widetilde{\alpha_{R} k_{F}}=30 \mathrm{meV}, \widetilde{\beta_{\mathrm{SO}}}=0 \mathrm{meV}$ ), where $B_{c}$ is far below the experimental data, indicating Rashba-type SOI alone cannot account for the observed large in-plane critical field.

In conclusion, we systematically investigate the transport properties of ultrathin crystalline $\mathrm{Pb}$ films at low temperatures and high magnetic fields. Under a parallel magnetic field, superconductivity protected by intrinsic Zeeman-type SOI survives at high fields far beyond the Pauli limit. We demonstrate that the interface between $\mathrm{Pb}$ film and $\mathrm{Si}$ substrate plays a key role in breaking in-plane inversion symmetry and hence inducing Zeeman-protected superconductivity in $\mathrm{Pb}$ films. Our work reveals that, in epitaxial heterostructures, the interface-modulated SOI has profound influence on the superconducting pairing and may provide a promising platform to study unconventional superconductivity [32].

We thank Ying Xing, Yanan Li, Jinglei Zhang, Youmin Zou, Jiezun Ke, Liang Li, Cheng Chen, and Yue Tang for help with transport measurements. This work was financially supported by the National Basic Research Program of China (Grants No. 2013CB934600, No. 2017YFA0303302, No. 2016YFA0301004, No. 2015CB921102, No. 2017YFA0303301, and No. 2017YFA0304600), the National Natural Science Foundation of China (Grants No. 11774008, No. 11534001, and No. 11674028), the Open Project Program of the Pulsed High Magnetic Field Facility (Grant No. PHMFF2015002) at the Huazhong University of Science and Technology, and the Key Research Program of the Chinese Academy of Sciences (Grant No. XDPB08-1). H.-W. L. also acknowledges support from the Fundamental Research Funds for the Central Universities.

Y. L. and Z.W. contributed equally to this work.

[1] Y. Guo, Y.-F. Zhang, X.-Y. Bao, T.-Z. Han, Z. Tang, L.-X. Zhang, W.-G. Zhu, E. G. Wang, Q. Niu, Z. Q. Qiu, J.-F. Jia, Z.-X. Zhao, and Q.-K. Xue, Superconductivity Modulated by Quantum Size Effects, Science 306, 1915 (2004).

[2] M. M. Ozer, J. R. Thompson, and H. H. Weitering, Hard Superconductivity of a Soft Metal in the Quantum Regime, Nat. Phys. 2, 173 (2006).

[3] D. Eom, S. Qin, M. Y. Chou, and C. K. Shih, Persistent Superconductivity in Ultrathin Pb Films: A Scanning Tunneling Spectroscopy Study, Phys. Rev. Lett. 96, 027005 (2006).

[4] S. Y. Qin, J. D. Kim, Q. Niu, and C. K. Shih, Superconductivity at the Two-Dimensional Limit, Science 324, 1314 (2009).

[5] T. Zhang, P. Cheng, W.-J. Li, Y.-J. Sun, G. Wang, X.-G. Zhu, K. He, L. Wang, X. Ma, X. Chen, Y. Wang, Y. Liu, H.-Q. Lin, J.-F. Jia, and Q.-K. Xue, Superconductivity in One-Atomic-Layer Metal Films Grown on Si(111), Nat. Phys. 6, 104 (2010).

[6] T. Uchihashi, P. Mishra, M. Aono, and T. Nakayama, Macroscopic Superconducting Current through a Silicon Surface Reconstruction with Indium Adatoms: Si(111)$\sqrt{7} \times \sqrt{3}-$ In, Phys. Rev. Lett. 107, 207001 (2011).

[7] M. Yamada, T. Hirahara, and S. Hasegawa, Magnetoresistance Measurements of a Superconducting Surface State of In-Induced and Pb-Induced Structures on Si(111), Phys. Rev. Lett. 110, 237001 (2013). 
[8] C. Brun, T. Cren, V. Cherkez, F. Debontridder, S. Pons, D. Fokin, M. C. Tringides, S. Bozhko, L. B. Ioffe, B. L. Altshuler, and D. Roditchev, Remarkable Effects of Disorder on Superconductivity of Single Atomic Layers of Lead on Silicon, Nat. Phys. 10, 444 (2014).

[9] H.-M. Zhang, Y. Sun, W. Li, J.-P. Peng, C.-L. Song, Y. Xing, Q. Zhang, J. Guan, Z. Li, Y. Zhao, S. Ji, L. Wang, K. He, X. Chen, L. Gu, L. Ling, M. Tian, L. Li, X. C. Xie, J. Liu et al., Detection of a Superconducting Phase in a Two-Atom Layer of Hexagonal Ga Film Grown on Semiconducting GaN (0001), Phys. Rev. Lett. 114, 107003 (2015).

[10] Y. Xing, H.-M. Zhang, H.-L. Fu, H. Liu, Y. Sun, J.-P. Peng, F. Wang, X. Lin, X.-C. Ma, Q.-K. Xue, J. Wang, and X. C. Xie, Quantum Griffiths Singularity of SuperconductorMetal Transition in Ga Thin Films, Science 350, 542 (2015).

[11] Q.-Y. Wang, Z. Li, W.-H. Zhang, Z.-C. Zhang, J.-S. Zhang, W. Li, H. Ding, Y.-B. Ou, P. Deng, K. Chang, J. Wen, C.-L. Song, K. He, J.-F. Jia, S.-H. Ji, Y.-Y. Wang, L.-L. Wang, X. Chen, X.-C. Ma, and Q.-K. Xue, Interface-Induced HighTemperature Superconductivity in Single Unit-Cell FeSe Films on $\mathrm{SrTiO}_{3}$, Chin. Phys. Lett. 29, 037402 (2012).

[12] W.-H. Zhang, Y. Sun, J.-S. Zhang, F.-S. Li, M.-H. Guo, Y.-F. Zhao, H.-M. Zhang, J.-P. Peng, Y. Xing, H.-C. Wang, T. Fujita, A. Hirata, Z. Li, H. Ding, C.-J. Tang, M. Wang, Q.-Y. Wang, K. He, S.-H. Ji, X. Chen et al., Direct Observation of High-Temperature Superconductivity in One-Unit-Cell FeSe Films, Chin. Phys. Lett. 31, 017401 (2014).

[13] A. M. Clogston, Upper Limit for the Critical Field in Hard Superconductors, Phys. Rev. Lett. 9, 266 (1962).

[14] B. S. Chandrasekhar, A Note on the Maximum Critical Field of High-Field Superconductors, Appl. Phys. Lett. 1, 7 (1962).

[15] D. Aoki, A. Huxley, E. Ressouche, D. Braithwaite, J. Flouquet, J.-P. Brison, E. Lhotel, and C. Paulsen, Coexistence of Superconductivity and Ferromagnetism in URhGe, Nature (London) 413, 613 (2001).

[16] R. A. Klemm, A. Luther, and M. R. Beasley, Theory of Upper Critical-Field in Layered Superconductors, Phys. Rev. B 12, 877 (1975).

[17] P. M. Tedrow and R. Meservey, Critical Magnetic Field of Very Thin Superconducting Aluminum Films, Phys. Rev. B 25, 171 (1982).

[18] This statement was first put forward in Refs. [19] and [20]. See Part III of Ref. [21] for further discussion on this point.

[19] J. M. Lu, O. Zheliuk, I. Leermakers, N. F. Q. Yuan, U. Zeitler, K. T. Law, and J. T. Ye, Evidence for TwoDimensional Ising Superconductivity in Gated $\mathrm{MoS}_{2}$, Science 350, 1353 (2015).
[20] Y. Saito, Y. Nakamura, M. S. Bahramy, Y. Kohama, J. Ye, Y. Kasahara, Y. Nakagawa, M. Onga, M. Tokunaga, T. Nojima, Y. Yanase, and Y. Iwasa, Superconductivity Protected by Spin-Valley Locking in Ion-Gated $\mathrm{MoS}_{2}$, Nat. Phys. 12, 144 (2016).

[21] See Supplemental Material at http://link.aps.org/ supplemental/10.1103/PhysRevX.8.021002 for more details.

[22] Y. Saito, T. Nojima, and Y. Iwasa, Highly Crystalline 2D Superconductors, Nat. Rev. Mater. 2, 16094 (2016).

[23] X. Xi, Z. Wang, W. Zhao, J.-H. Park, K. T. Law, H. Berger, L. Forró, J. Shan, and K. F. Mak, Ising Pairing in Superconducting $\mathrm{NbSe}_{2}$ Atomic Layers, Nat. Phys. 12, 139 (2016).

[24] Y. Xing, K. Zhao, P. Shan, F. Zheng, Y. Zhang, H. Fu, Y. Liu, M. Tian, C. Xi, H. Liu, J. Feng, X. Lin, S. Ji, X. Chen, Q.-K. Xue, and J. Wang, Ising Superconductivity and Quantum Phase Transition in Macro-Size Monolayer $\mathrm{NbSe}_{2}$, Nano Lett. 17, 6802 (2017).

[25] N. R. Werthamer, E. Helfand, and P. C. Hohenberg, Temperature and Purity Dependence of the Superconducting Critical Field, $\mathrm{H}_{c 2}$. III. Electron Spin and Spin-Orbit Effects, Phys. Rev. 147, 295 (1966).

[26] K. Epstein, A. M. Goldman, and A. M. Kadin, VortexAntivortex Pair Dissociation in Two-Dimensional Superconductors, Phys. Rev. Lett. 47, 534 (1981).

[27] N. Reyren, S. Thiel, A. D. Caviglia, L. F. Kourkoutis, G. Hammerl, C. Richter, C. W. Schneider, T. Kopp, A. S. Rüetschi, D. Jaccard, M. Gabay, D. A. Muller, J. M. Triscone, and J. Mannhart, Superconducting Interfaces Between Insulating Oxides, Science 317, 1196 (2007).

[28] A. A. Abrikosov, L. P. Gorkov, and I. E. Dzialochinski, Methods of Quantum Field Theory in Statistical Physics (Prentice-Hall, Englewood Cliffs, NJ, 1963).

[29] T.-L. Chan, C. Z. Wang, M. Hupalo, M. C. Tringides, Z.-Y. Lu, and K. M. Ho, First-Principles Studies of Structures and Stabilities of $\mathrm{Pb} / \mathrm{Si}(111)$, Phys. Rev. B 68, 045410 (2003).

[30] X. Y. Ren, H.-J. Kim, S. Yi, Y. Jia, and J.-H. Cho, SpinOrbit Coupling Effects on the Stability of Two Competing Structures in $\mathrm{Pb} / \mathrm{Si}(111)$ and $\mathrm{Pb} / \mathrm{Ge}(111)$, Phys. Rev. B 94, 075436 (2016).

[31] C. Brand, S. Muff, M. Fanciulli, H. Pfnür, M. C. Tringides, J. H. Dil, and C. Tegenkamp, Spin-Resolved Band Structure of a Densely Packed Pb Monolayer on Si(111), Phys. Rev. B 96, 035432 (2017).

[32] R. Wakatsuki and K. T. Law, Proximity Effect and Ising Superconductivity in Superconductor/Transition Metal Dichalcogenide Heterostructures, arXiv:1604.04898. 